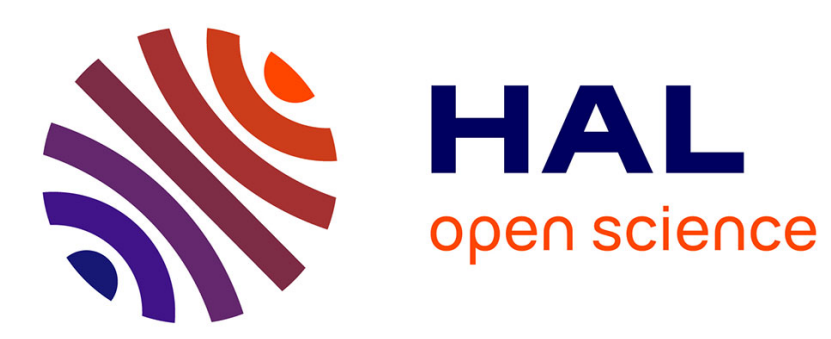

\title{
Nonlinear evolution of the zigzag instability in stratified fluids: A shortcut on the route to dissipation
}

\author{
Axel Deloncle, Paul Billant, Jean-Marc Chomaz
}

\section{To cite this version:}

Axel Deloncle, Paul Billant, Jean-Marc Chomaz. Nonlinear evolution of the zigzag instability in stratified fluids: A shortcut on the route to dissipation. Journal of Fluid Mechanics, 2008, 599 (mars), pp.229-239. 10.1017/s0022112007000109 . hal-01022803

\section{HAL Id: hal-01022803 \\ https://hal-polytechnique.archives-ouvertes.fr/hal-01022803}

Submitted on 21 Jul 2014

HAL is a multi-disciplinary open access archive for the deposit and dissemination of scientific research documents, whether they are published or not. The documents may come from teaching and research institutions in France or abroad, or from public or private research centers.
L'archive ouverte pluridisciplinaire HAL, est destinée au dépôt et à la diffusion de documents scientifiques de niveau recherche, publiés ou non, émanant des établissements d'enseignement et de recherche français ou étrangers, des laboratoires publics ou privés. 


\title{
Nonlinear evolution of the zigzag instability in stratified fluids: a shortcut on the route to dissipation
}

\author{
AXEL DELONCLE, PAUL BILLANT \\ AND JEAN-MARC CHOMAZ \\ LadHyX, CNRS, Ecole Polytechnique, 91128 Palaiseau Cedex, France \\ axel.deloncle@ladhyx.polytechnique.fr
}

(Received 19 July 2007 and in revised form 22 November 2007)

We present high-resolution direct numerical simulations of the nonlinear evolution of a pair of counter-rotating vertical vortices in a stratified fluid for various high Reynolds numbers $R e$ and low Froude numbers $F_{h}$. The vortices are bent by the zigzag instability producing high vertical shear. There is no nonlinear saturation so that the exponential growth is stopped only when the viscous dissipation by vertical shear is of the same order as the horizontal transport, i.e. when $Z_{\max }^{h} / R e=O(1)$ where $Z_{\max }^{h}$ is the maximum horizontal enstrophy non-dimensionalized by the vortex turnover frequency. The zigzag instability therefore directly transfers the energy from large scales to the small dissipative vertical scales. However, for high Reynolds number, the vertical shear created by the zigzag instability is so intense that the minimum local Richardson number $R i$ decreases below a threshold of around 1/4 and small-scale Kelvin-Helmholtz instabilities develop. We show that this can only occur when $\operatorname{Re} F_{h}^{2}$ is above a threshold estimated as 340. Movies are available with the online version of the paper.

\section{Introduction}

In a strongly stratified fluid, pairs of counter-rotating and co-rotating vertical vortices are subject to the zigzag instability (Billant \& Chomaz 2000a; Otheguy, Chomaz \& Billant 2006). This linear three-dimensional instability bends the vortices with a most unstable vertical wavelength scaling with the buoyancy length scale $b F_{h}$, where $b$ is the distance between the two vortices and $F_{h}$ the horizontal Froude number $\left(F_{h}=\Gamma / 2 \pi a^{2} N\right.$ with $a$ the vortices core radius, $\Gamma$ their circulation and $N$ the Brunt-Väisälä frequency), and develops with a growth rate proportional to the strain $\Gamma / 2 \pi b^{2}$.

In the experiments of Billant \& Chomaz (2000a), the zigzag instability of a counterrotating vortex pair grows to a large amplitude and produces layers of pancake dipoles separated by regions of extreme vertical shear. However, in these experiments, the Reynolds number is relatively low whereas atmospheric and oceanic flows have high Reynolds numbers. We have therefore performed direct numerical simulations (DNS) of the zigzag instability of a pair of counter-rotating vortices at high Reynolds number in order to understand the nonlinear development of this linear instability and the resulting energy transfer. 


\section{Numerical method}

A pseudo-spectral method with periodic boundary conditions is used to solve the nonlinear incompressible Navier-Stokes equations under the Boussinesq approximation. Time advancement is carried out with the classical fourth-order Runge-Kutta scheme for the nonlinear term and exact integration for the viscous and diffusive terms (see Vincent \& Meneguzzi 1991 for details). Most of the aliasing is removed by truncating $9 / 10$ of the modes along each direction.

The velocity field of the DNS is initialized as

$$
\boldsymbol{U}(x, y, z, t=0)=\boldsymbol{U}_{2 D}(x, y)+\varepsilon \cos \left(k_{z \max } z\right) \boldsymbol{U}_{p}(x, y),
$$

where $\boldsymbol{U}_{2 D}$ is a two-dimensional flow representing a quasi-steady vortex pair, $\boldsymbol{U}_{p}$ its most unstable three-dimensional zigzag eigenmode with vertical wavenumber $k_{z \max }, \varepsilon$ a chosen small amplitude, $z$ the vertical coordinate and $(x, y)$ the horizontal Cartesian coordinates.

The flow $\boldsymbol{U}_{2 D}$ is obtained from a two-dimensional nonlinear simulation initialized by a pair of counter-rotating Gaussian vortices of circulation $\pm \Gamma$, radius $a$ and separated by a distance $b=2.5 a$, corresponding to the vertical vorticity

$$
\omega_{z}(x, y)=\Gamma / \pi a^{2}\left[\exp \left(-\left(x^{2}+y^{2}\right) / a^{2}\right)-\exp \left(-\left(x^{2}+(y+b)^{2}\right) / a^{2}\right] .\right.
$$

As time evolves, each vortex rapidly adapts to the strain exerted by the other one so that a quasi-steady state $\boldsymbol{U}_{2 D}$ is quickly reached (Sipp, Jacquin \& Cossu 2000). The initial state is presented at $t=0$ in figure $1(a)$.

The Reynolds number $R e$ and the Froude number $F_{h}$ are based on the initial conditions:

$$
R e=\frac{\Gamma}{2 \pi v}, \quad F_{h}=\frac{\Gamma}{2 \pi a^{2} N},
$$

where $v$ is the kinematic viscosity and $N$ the Brunt-Väisälä frequency. The Schmidt number $S c=v / D$, where $D$ is the mass diffusivity, is set to unity in all runs. In what follows, the time unit is taken as $2 \pi a^{2} / \Gamma$, the length unit as $a$ and the density unit as $a\left|\mathrm{~d} \rho_{0} / \mathrm{d} z\right|$ where $\rho_{0}(z)$ is a reference density.

The flow $\boldsymbol{U}_{p}$ is the most unstable three-dimensional perturbation of $\boldsymbol{U}_{2 D}$ determined by a numerical linear stability analysis for each Froude number. Since $F_{h}<1$, it corresponds to the eigenmode of the zigzag instability with a most amplified wavenumber $k_{z \max }$ proportional to $F_{h}$, in agreement with the linear theory of the zigzag instability (Billant \& Chomaz 2000b).

The parameters of each run are summarized in table 1 . The height of the computational domain $L_{z}$ is adjusted so as to have only one wavelength in the box. The horizontal size of the box $L_{x}=L_{y}=10.5$ is taken sufficiently large compared to the distance between the two vortices in order to have negligible effects of the periodic boundary conditions. Furthermore, several additional cases were run, changing the domain size and/or the numerical resolution, in order to test the accuracy and robustness of the results presented. The results of these additional runs were consistent with those presented in the following, and so will not be discussed.

\section{Qualitative behaviour}

The zigzag instability exhibits two different nonlinear evolutions depending on the Reynolds and Froude numbers. Figure 1 shows the temporal evolution of the vertical vorticity isosurfaces for these two distinct behaviours: figure 1(a) for run 
(a)
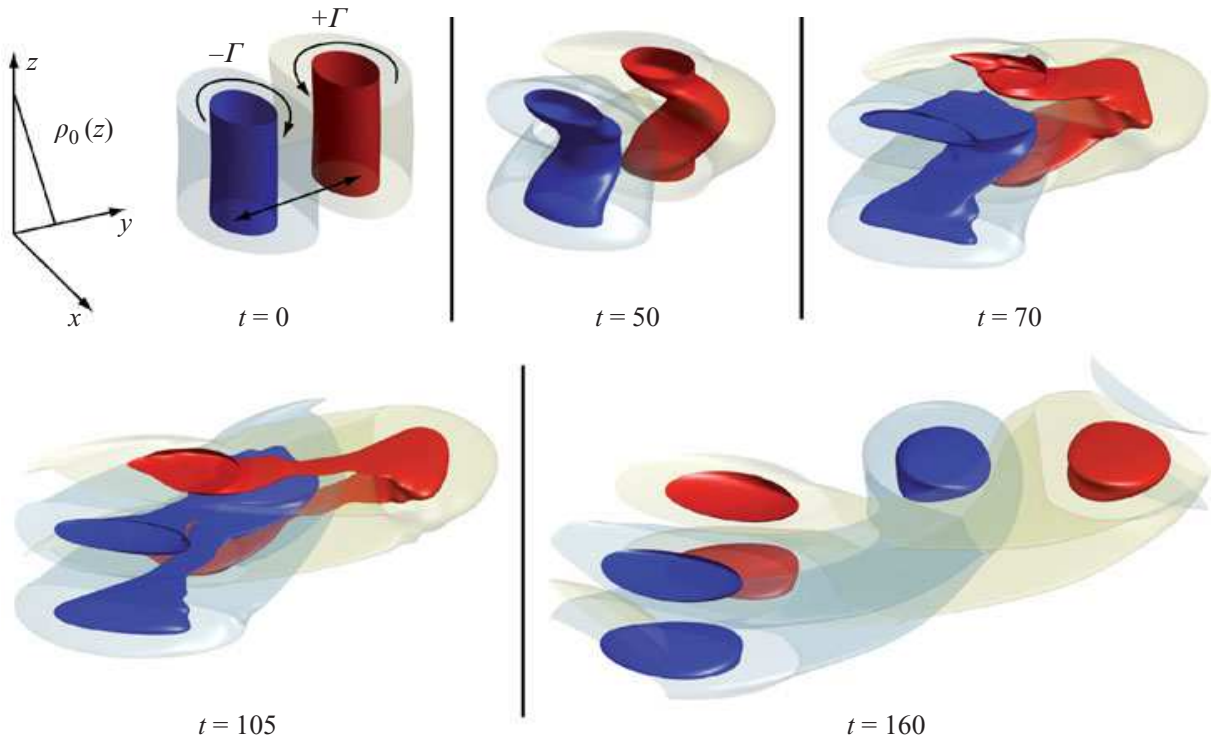

(b)
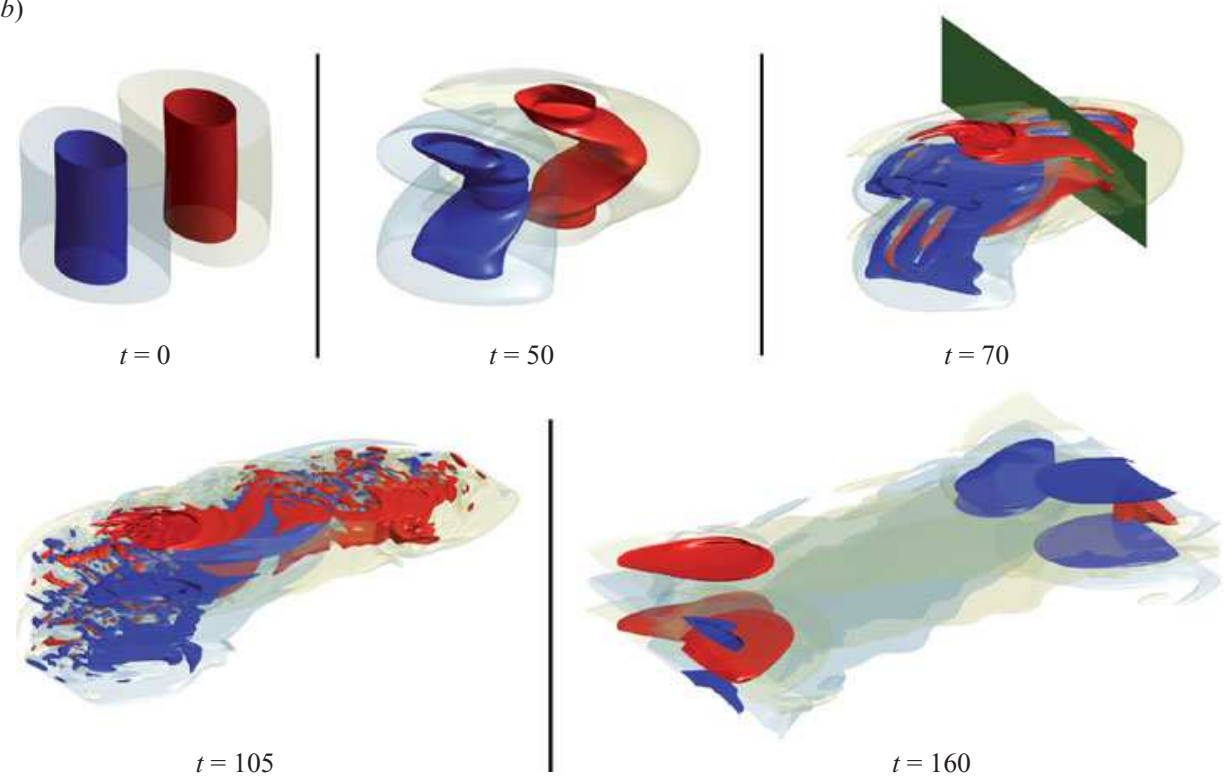

FiguRE 1. Vertical vorticity isosurfaces of the simulations $(a)$ F0.6R1 $\left(F_{h}=0.66, R e=1060\right)$ and $(b)$ F0.6R3 $\left(F_{h}=0.66, R e=3180\right)$. Red and deep blue contours represent respectively plus and minus $60 \%$ of $\left\langle\omega_{z}^{\max }\right\rangle_{z}$, the vertical average of the maximum vertical vorticity in each horizontal plane. Transparent yellowish and bluish isosurfaces are the same for a $10 \%$ level. The green plane shown at $t=70$ in $(b)$ indicates the position of the vertical cross-sections presented in figures 5 and 7. The corresponding movies are available with the online version of the paper.

F0.6R1 $\left(F_{h}=0.66, R e=1060\right)$ and figure $1(b)$ for run F0.6R3 $\left(F_{h}=0.66, R e=3180\right)$. Corresponding movies are available with the online version of the paper. At the beginning $(t=0)$, the two vortices are straight along the vertical since the 


$\begin{array}{lcrrrccc}\text { Run } & F_{h} & R e & R=R e F_{h}^{2} & k_{z} \max & L_{x} \times L_{y} \times L_{z} & N_{x} \times N_{y} \times N_{z} & \delta_{t} \\ \text { F0.6R1 } & 0.66 & 1060 & 460 & 2.2 & 10.5 \times 10.5 \times 2.85 & 256 \times 256 \times 64 & 0.04 \\ \text { F0.6R3 } & 0.66 & 3180 & 1380 & 2.2 & 10.5 \times 10.5 \times 2.85 & 384 \times 384 \times 96 & 0.02 \\ \text { F0.6R6 } & 0.66 & 6360 & 2770 & 2.2 & 10.5 \times 10.5 \times 2.85 & 768 \times 768 \times 192 & 0.01 \\ \text { F0.3R4 } & 0.33 & 4240 & 460 & 4.4 & 10.5 \times 10.5 \times 1.425 & 512 \times 512 \times 72 & 0.02 \\ \text { F0.3R12 } & 0.33 & 12720 & 1380 & 4.4 & 10.5 \times 10.5 \times 1.425 & 1440 \times 1440 \times 192 & 0.004\end{array}$

TABLE 1. Overview of the physical and numerical parameters of the main simulations described in the paper. The number of nodes in the $x$-, $y$ - and $z$-directions are denoted, respectively, $N_{x}, N_{y}$ and $N_{z}$. The time step is $\delta_{t}$. For all runs $S c=1, a=1$ and $b=2.5$.
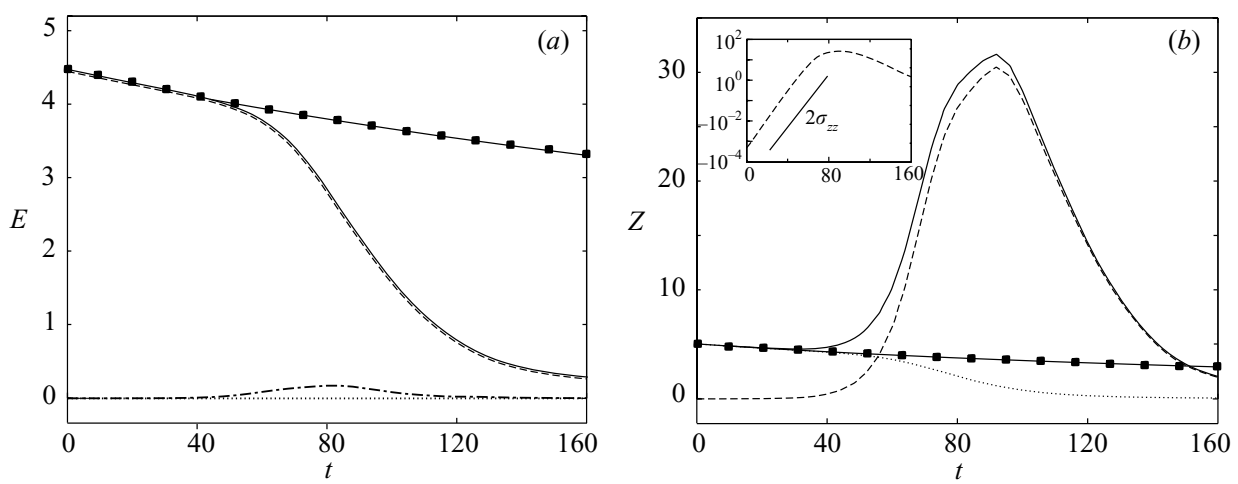

Figure 2. (a) Energy and $(b)$ enstrophy evolutions of the run F0.6R1 $\left(F_{h}=0.66, R e=1060\right)$. In $(a)$, the total kinetic energy $E_{k}^{T}$ is plotted (solid line) as well as its horizontal $E_{k}^{h}$ (dashed line) and vertical $E_{\kappa}^{v}$ (dotted line) components. Potential energy $E_{p}$ (dash-dot line) is always negligible compared to the total kinetic energy $E_{k}^{T}$. The evolution of total kinetic energy for

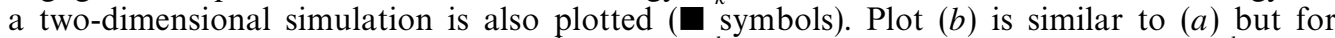
the enstrophy $Z$. A semi-log plot of $Z^{h}$ (i.e. $\log Z^{h}$ versus $t$ ) (inset of $b$ ) shows that $Z^{h}$ grows exponentially in time until $t \sim 70$ at a rate of $2 \sigma_{z z}$ where $\sigma_{z z}$ is the growth rate of the zigzag instability.

three-dimensional perturbation is very small. By $t=50$, the vortices are bent as a whole by the zigzag instability. Subsequently, the amplitude of the bending deformations for run F0.6R 1 continues to grow dramatically without apparent saturation $(t=70$, $t=105$ ) as observed experimentally (Billant \& Chomaz 2000a). This eventually produces decorrelated thin horizontal layers $(t=160)$, but at that time a strong dissipation has occurred since the isosurfaces shown are only $12 \%$ of the initial values. A distinctly different behaviour is observed at the intermediate times $(t=70$, $t=105$ ) for a higher Reynolds number (run F0.6R3, figure $1 b$ ). Small-scale threedimensional structures develop in the highly sheared region of the vortices so that the vortices break down. However, at late time $(t=160)$, smooth decorrelated layers are seen again like those in figure $1(a)$.

\section{Energy and enstrophy analysis}

Figure 2(a) shows the temporal evolution of the total kinetic energy $E_{k}^{T}=\langle\boldsymbol{u} \cdot \boldsymbol{u}\rangle / 2$ (where $\boldsymbol{u}$ is the velocity and $\langle$.$\rangle denotes the volume integral per unit vertical length:$ $\left.\langle\rangle=.\left(1 / L_{z}\right) \int_{V} \cdot \mathrm{d} V\right)$ as well as its horizontal $E_{k}^{h}$ and vertical $E_{k}^{v}$ components for a typical run: F0.6R1. Almost all the kinetic energy is contained in the horizontal 

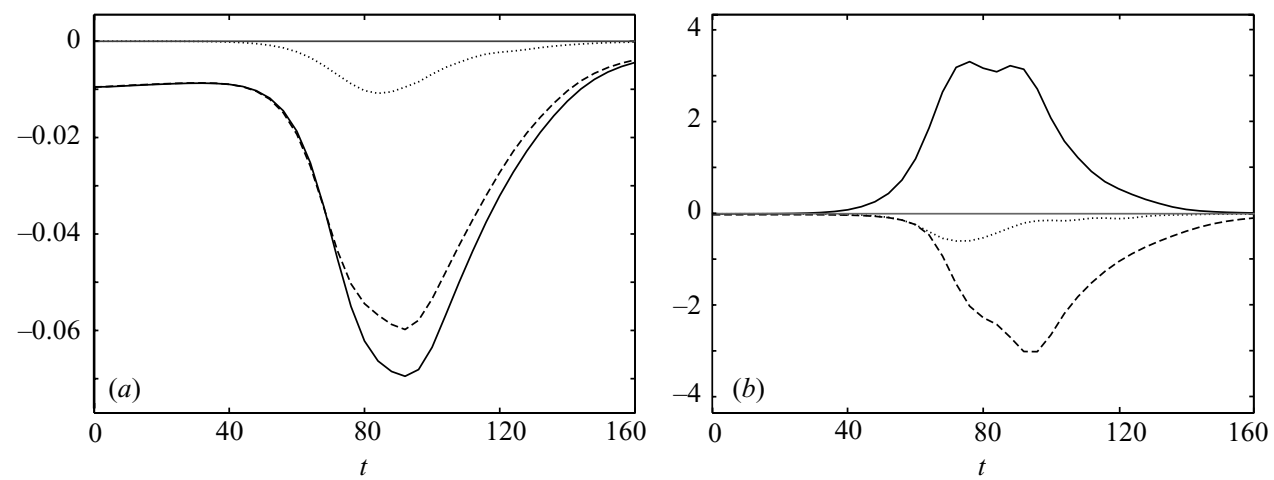

FIGURE 3. Evolutions of $(a)$ energy dissipation and $(b)$ enstrophy sources for run F0.6R1 $\left(F_{h}=0.66, R e=1060\right)$. (a) The energy dissipation $\mathrm{d}\left(E_{k}^{T}+E_{p}\right) / \mathrm{d} t$ (solid line) is mainly caused by viscous effects (dashed line) which are much more important than the potential energy dissipation (dotted line). (b) Evolution of the enstrophy sources: stretching (solid line), baroclinic (dotted line) and viscous (dashed line) terms.

motion which confirms that vertical displacements are inhibited by stratification. Up to $t \sim 40$, the evolution of the total kinetic energy (solid line) is identical to that of a purely two-dimensional flow without the zigzag instability ( $\mathbf{\square}$ symbols). Then a strong decrease of kinetic energy is observed when the zigzag instability develops. The potential energy $E_{p}=\left\langle\rho^{2}\right\rangle / 2 F_{h}^{2}$ where $\rho$ is the non-dimensional density perturbation (see e.g. Gill 1982 for a detailed discussion of potential energy) is also shown in figure 2(a) (dash-dot line) but it always remains negligible compared to the kinetic energy.

Figure $2(b)$ presents the temporal evolution of the total enstrophy $Z^{T}=\langle\boldsymbol{\omega} \cdot \boldsymbol{\omega}\rangle / 2$ where $\omega$ is the vorticity, and its horizontal $Z^{h}$ and vertical $Z^{v}$ components. The total enstrophy first decreases as in the two-dimensional case up to $t \sim 40$, then strongly increases up to $t \sim 90$ and eventually relaxes. The vertical enstrophy decreases steadily whereas the horizontal enstrophy grows exponentially owing to the development of the zigzag instability from $t=0$ up to $t \sim 70$ as demonstrated by the inset graph in figure $2(b)$. It then saturates around $t \sim 80$ when the decrease of energy is the fastest.

These behaviours can be understood by looking at the total energy equation in non-dimensional form:

$$
\frac{\mathrm{d}\left(E_{k}^{T}+E_{p}\right)}{\mathrm{d} t}=-2 \frac{Z^{T}}{\operatorname{Re}}-\frac{1}{\operatorname{ReSc}} \frac{\langle\nabla \rho \cdot \nabla \rho\rangle}{F_{h}^{2}} .
$$

Figure 3(a) presents the temporal evolution of the two dissipative processes of (4.1): we see that the kinetic energy dissipation (first term on the right-hand side of (4.1)) is largely dominant over the potential energy dissipation (second term on the right-hand side of (4.1)). The kinetic energy dissipation is directly related to the total enstrophy whose equation in non-dimensional form is

$$
\frac{\mathrm{d} Z^{T}}{\mathrm{~d} t}=\langle\boldsymbol{\omega} \cdot(\boldsymbol{\omega} \cdot \nabla \boldsymbol{u})\rangle-\frac{1}{F_{h}^{2}}\left\langle\boldsymbol{\omega} \cdot \nabla \times\left(\rho \boldsymbol{e}_{z}\right)\right\rangle-\frac{1}{R e}\langle\nabla \omega: \nabla \omega\rangle .
$$

The three terms on the right-hand side of (4.2) correspond respectively to stretching, baroclinic and viscous effects. The temporal evolution of these three terms in figure $3(b)$ shows that the only source of enstrophy is the stretching term. This stretching effect comes from the high shear due to the bending of the vortices by 

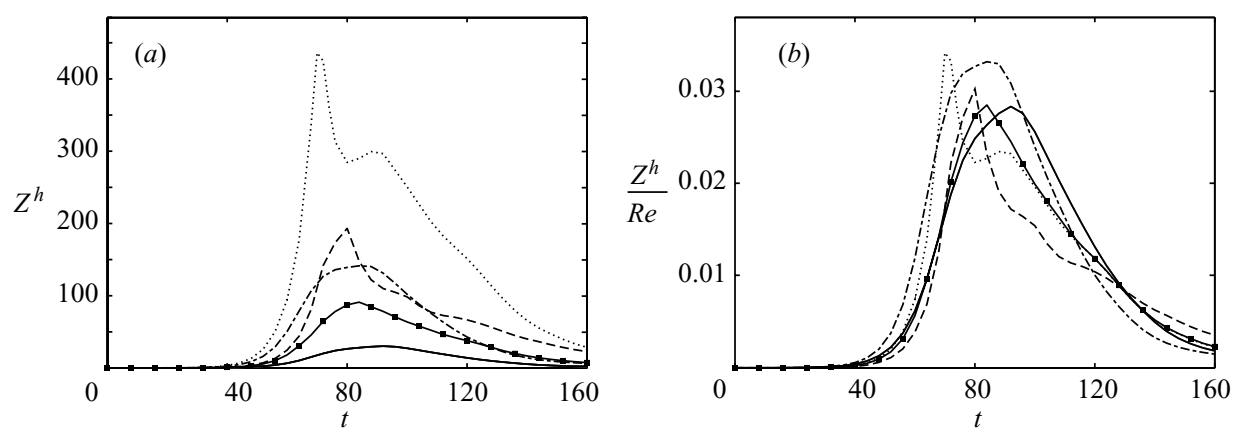

FIGURE 4. Evolutions of $(a)$ the horizontal enstrophy $Z^{h}$ and $(b)$ the rescaled horizontal enstrophy $Z^{h} / R e$ for different runs: F0.6R1 ( $R e=1060$, solid line), F0.6R3 $(R e=3180$, symbols), F0.3R4 ( $R e=4240$, dash-dot line), F0.6R6 $(R e=6360$, dashed line) and F0.3R12 $(R e=12720$, dotted line).

the zigzag instability. It results in an exponential growth of horizontal enstrophy $Z^{h}$ which becomes so large that $Z^{T} \sim Z^{h}$. It is then high enough to dissipate the initial kinetic energy and to saturate the development of the zigzag instability.

This physical mechanism implies that the maximum value of horizontal enstrophy $Z_{\max }^{h}$ is reached when the dissipation term in (4.1) induces an order-one variation of the energy: $Z_{\max }^{T} / R e \sim Z_{\max }^{h} / R e=O(1)$. This is confirmed by figure 4 which shows the evolution of horizontal enstrophy for several simulations covering a range of Reynolds numbers from $R e=1060$ to $R e=12720$. As predicted, the maximum value of horizontal enstrophy (figure $4 a$ ) increases with the Reynolds number. There is an abrupt decrease for the runs with highest Reynolds numbers (F0.6R6 and F0.3R12) because of the intense development of the small-scale instability. However, the different curves collapse well when $Z_{h}$ is rescaled by the Reynolds number (figure $4 b$ ).

The zigzag instability is therefore a shortcut on the route to dissipation since it produces a direct transfer of energy from the large scales to the small dissipative vertical scales. Horizontal enstrophy grows exponentially due to the zigzag instability until the vertical shear has reached the dissipative scales. This mechanism of stretching of the horizontal enstrophy can be seen as the stratified equivalent of the stretching of vortices in homogeneous flows. It eventually leads to a flow dominated by viscous effects.

\section{Kelvin-Helmholtz instability}

Figure 1(b) corresponding to run F0.6R3 shows the development of small-scale structures in the highly sheared region of the vortices. To address their origin, several authors (Riley \& deBruynKops 2003; Laval, McWilliams \& Dubrulle 2003; Waite \& Bartello 2004) have proposed considering the local Richardson number $R i$ :

$$
R i=\frac{1+(\partial \rho / \partial z)}{F_{h}^{2}\left[(\partial u / \partial z)^{2}+(\partial v / \partial z)^{2}\right]},
$$

where $1+\partial \rho / \partial z$ is the non-dimensional vertical gradient of the total density. The presence of $F_{h}$ in (5.1) comes from the non-dimensionalization (see $\S 2$ ).

Figure 5 shows a time series of the density perturbations and the local Richardson number in a vertical cross-section (whose position is indicated in figure $1 b$ at $t=70$ ). We clearly see (figure $5 a$ ) small-scale billows resembling Kelvin-Helmholtz rolls at 
(a)

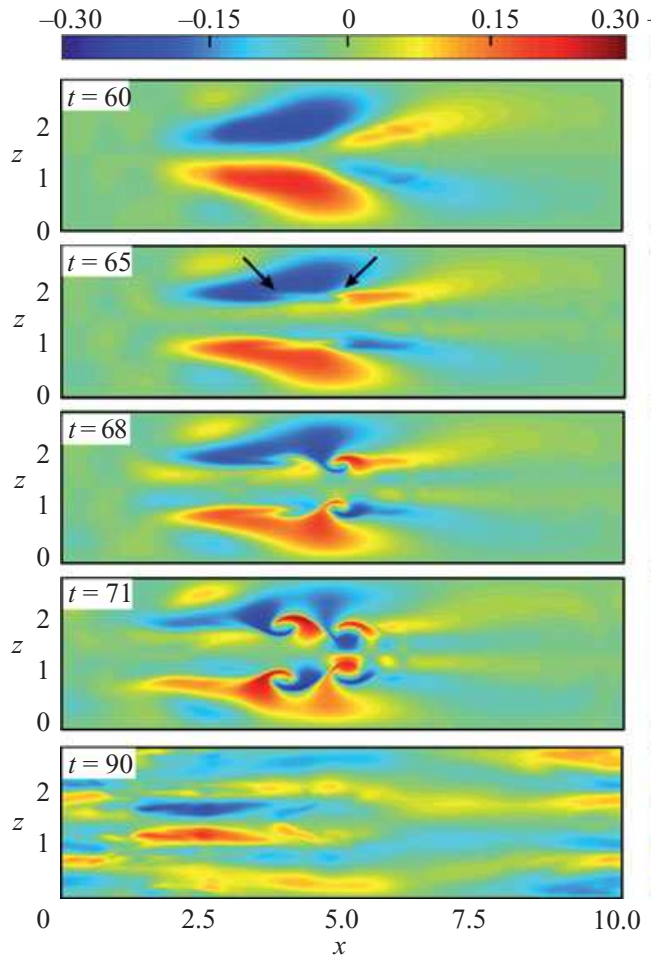

(b)

$\begin{array}{llll}0.25 & 0 & 0.25 & 0.50\end{array}$
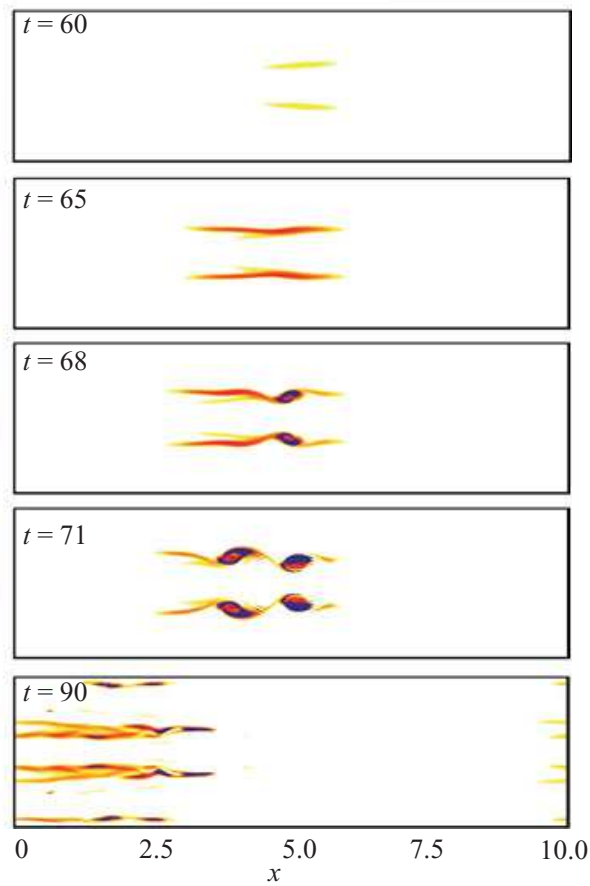

FIGURE 5. Time evolution of a vertical cross-section of $(a)$ density perturbations and (b) Richardson number of the run F0.6R3 $\left(F_{h}=0.66, R e=3180\right)$. The position of the cross-section is indicated in figure $1(b)$. The Kelvin-Helmholtz instability appears at $t=65$ in regions where $R i<1 / 4$ and produces a roll-up as shown at $t=68$ and $t=71$ by the regions where $R i$ is negative. The billows break down into irregular structures by $t=90$.

$t=68$ and $t=71$ but they are already incipient at $t=65$ as marked by the arrows. The roll-up occurs in the regions where the Richardson number (figure $5 b$ ) is lower than $1 / 4$, demonstrating that its origin is the Kelvin-Helmholtz instability (Miles 1961; Howard 1961). This instability is not of Rayleigh-Taylor (gravitational) type since the Richardson number is positive just before its onset at $t=65$ and becomes negative only in regions where the roll-up has occurred.

Following Riley \& deBruynKops (2003), the presence or absence of the KelvinHelmholtz instability for a given set of parameters $\left(F_{h}, R e\right)$ can be predicted using the Richardson criterion $R i<1 / 4$ and the scaling $Z_{\text {max }}^{h} \propto R e$. Indeed the non-dimensional local vertical shear $(\partial u / \partial z)^{2}+(\partial v / \partial z)^{2}$ can be estimated from the total vertical shear $S^{2}=\left\langle(\partial u / \partial z)^{2}+(\partial v / \partial z)^{2}\right\rangle$. This quantity is directly related to the total horizontal enstrophy $S^{2} \sim 2 Z^{h}$ when $F_{h} \ll 1$ since the vertical velocity is then very small and vertical derivatives are high (Billant \& Chomaz 2001). Therefore, the minimum Richardson number is

$$
R i_{\text {min }} \sim \frac{1}{S_{\max }^{2} F_{h}^{2}} \sim \frac{1}{2 Z_{\max }^{h} F_{h}^{2}} \propto \frac{1}{R e F_{h}^{2}},
$$

where we have assumed that the total density gradient is of the order of the ambient density gradient, i.e. $1+\partial \rho / \partial z \sim 1$. 


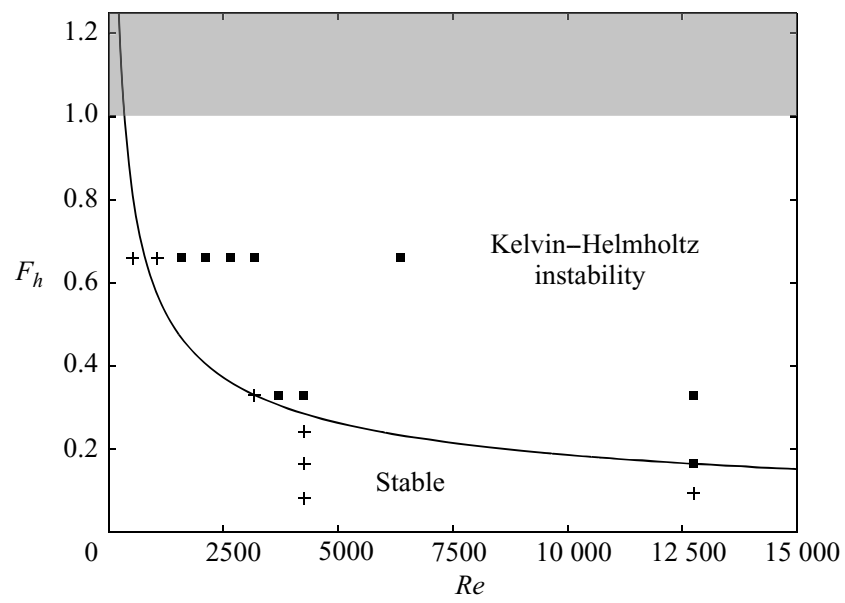

FIGURE 6. Summary of all the runs in the $F_{h}-R e$ plane. The symbol $\mathbf{\square}$ indicates a run in which Kelvin-Helmholtz instabilities are observed; otherwise a + symbol is used. The criterion for the occurrence of Kelvin-Helmholtz instability is the appearance of vertical roll-up. Note that additional runs not listed in table 1 have been performed in order to cover the $F_{h}-R e$ plane. The solid line corresponds to the criterion $R=\operatorname{Re} F_{h}^{2}=340$. In the shaded region $\left(F_{h}>1\right)$ the zigzag instability is damped.

Therefore, we expect the Kelvin-Helmholtz instability when $R=R e F_{h}^{2}$ is large. This is confirmed in figure 6 which summarizes all the runs in the parameters space $\left(F_{h}\right.$, $R e$ ). We see that the threshold $R \sim 340$, determined empirically, discriminates quite well the runs where the Kelvin-Helmholtz instability has been observed from those where no such instability occurs (the threshold is slightly underestimated at small $R e$ since the viscous dissipation of the base flow and of the perturbations is no longer small whereas it is neglected in the reasoning above).

Furthermore, the runs F0.6R3 and F0.6R6 shown in figure 7 have nearly identical roll-up, indicating that the wavelength of the Kelvin-Helmholtz instability $\lambda_{K H}$ is independent of the Reynolds number $R e$. On the contrary, it is approximately proportional to the Froude number: $\lambda_{K H} \propto F_{h}$, as seen by comparing run F0.6R3 $\left(F_{h}=0.66, \lambda_{K H} \sim 1\right)$ and run F0.3R4 $\left(F_{h}=0.33, \lambda_{K H} \sim 0.55\right)$ in figure 7 . These observations are consistent with the Richardson number criterion since the non-dimensional vertical shear $S_{c}$ at onset of the Kelvin-Helmholtz instability is $S_{c}^{2} \propto 1 / F_{h}^{2} R i_{c}$ where $R i_{c}=1 / 4$ is the critical $R i$. Since the wavelength of the KelvinHelmholtz instability scales with the shear thickness, i.e. $\lambda_{K H} \propto 1 / S$, we deduce that $\lambda_{K H} \propto F_{h}$ at the instability onset in agreement with figure 7 .

Subsequently, the Kelvin-Helmholtz billows break down into small scales but disappear quite rapidly (figure 5). However, with much higher Reynolds numbers as in real geophysical flows, we would expect that the small scales created by the Kelvin-Helmholtz billows should evolve for a longer time before being dissipated and thus should play a more important role in the dynamics of the flow.

\section{Conclusion and perspectives}

We have performed direct numerical simulations of the nonlinear development of the zigzag instability of a pair of counter-rotating vertical vortices in a stratified fluid for various values of Reynolds number $R e$ and Froude number $F_{h}$. We have observed 

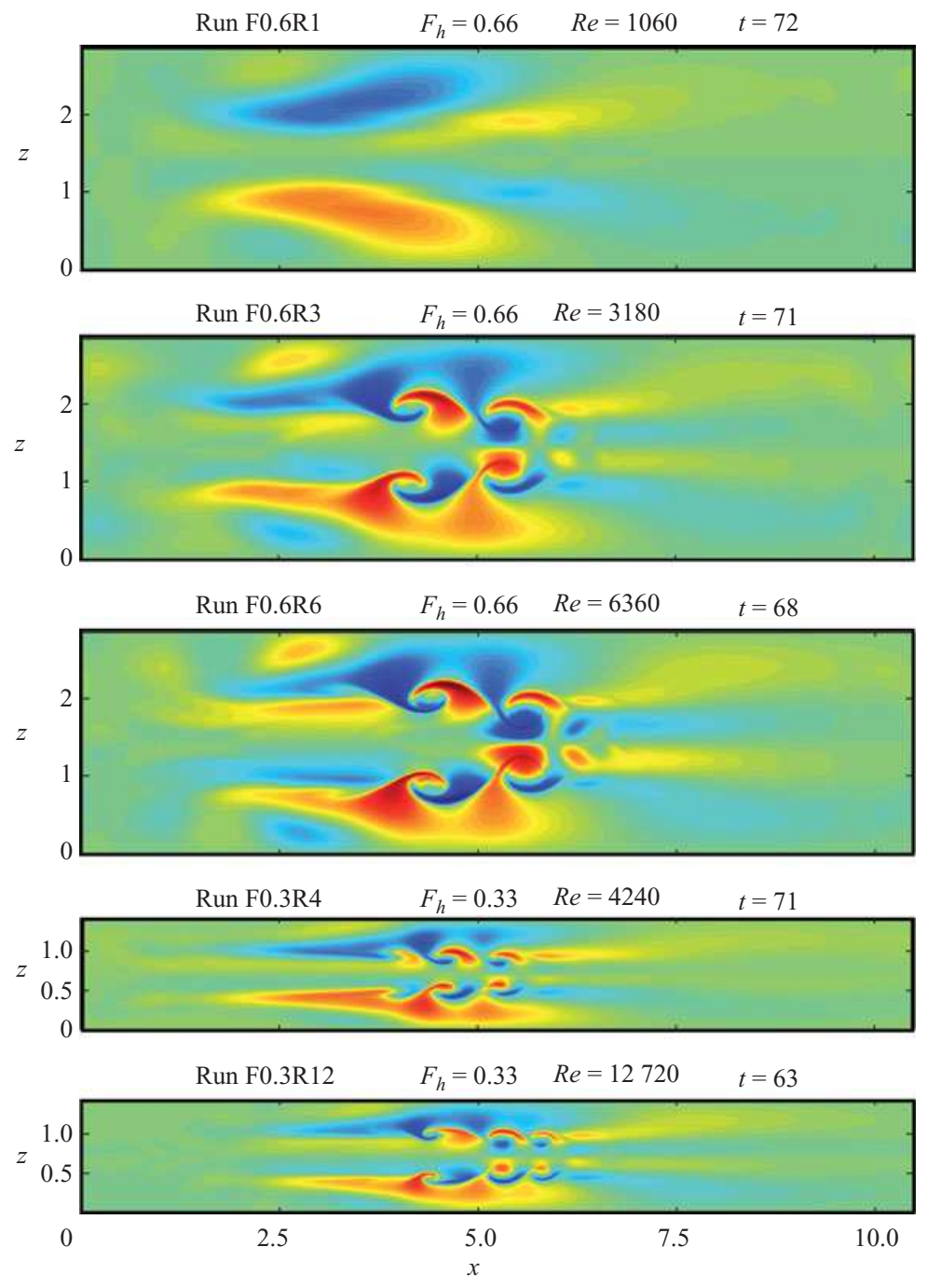

FiguRE 7. Vertical cross-sections of density perturbations for different simulations. The exact time of the cross-section is approximately the same but is adjusted for each simulation to show fully developed Kelvin-Helmholtz billows if any can be observed.

that the zigzag instability develops exponentially without saturation by nonlinear inertial effects. Thus, the vortices are distorted in an extreme manner, creating high vertical shear, i.e. horizontal vorticity. The exponential growth of the instability is only stopped when the vertical shear is such that vertical viscous effects become of the same order as the horizontal transport, i.e. when the maximum non-dimensional horizontal enstrophy is such that $Z_{\max }^{h} / R e=O(1)$. The maximum in time of the horizontal enstrophy observed in all simulations agrees with this scaling law. The subsequent evolution of the flow is dominated by vertical viscous effects.

We have also shown that the intense vertical shear created by the zigzag instability leads to Kelvin-Helmholtz instabilities for large values of $R=\operatorname{Re} F_{h}^{2} \gtrsim 340$, which allow for $R i_{\min }<1 / 4$ since $R i_{\min } \propto 1 / R e F_{h}^{2}$ following the arguments of Riley \& deBruynKops (2003). 
As pointed out by Riley \& deBruynKops (2003), the appearance of KelvinHelmholtz instabilities agrees to some extent with the suggestion of Lilly (1983) that vertical shear should increase in time and lead to Kelvin-Helmholtz instabilities. However, Lilly's suggestion is based on the hypothesis that a strongly stratified flow is a stack of two-dimensional flows without vertical coupling. In this case, a mean vertical shear increases algebraically in time if it is initially non-zero. In contrast, we have shown that the vertical shear arises spontaneously and grows exponentially in time because of the zigzag instability. Kelvin-Helmholtz instabilities are therefore expected to develop much faster than speculated by Lilly (1983).

Recent work has demonstrated that the zigzag instability is a generic linear instability mechanism that applies to many vorticity distributions such as a pair of counter-rotating vortices (Billant \& Chomaz 2000a), a pair of co-rotating vortices (Otheguy et al. 2006), an elliptic vortex with background strain (Billant, Dritschel \& Chomaz 2006), or vortex arrays (Deloncle, Billant \& Chomaz 2008). If we try to generalize the present results, pertaining to an idealized case, to more complex stratified flows and stratified turbulence, we may speculate that any flow with initial small horizontal and vertical Froude numbers should exhibit a series of instabilities similar to the one presented here. The energy would be first transferred from large to small vertical scales by the zigzag instability and then jump from large to small horizontal scales by the Kelvin-Helmholtz instability. These small vertical and horizontal scales are both of the order of the Ozmidov scale. This direct transfer to the Ozmidov scale might be an alternative to the stratified turbulent cascade proposed by Lindborg (2006) and Brethouwer et al. (2007).

After completion of this work, we became aware of a related study by Waite \& Smolarkiewicz (2007). In their large-eddy simulations (LES) at large Schmidt number, they study the nonlinear evolution of the zigzag instability for a Lamb-Chaplygin dipole. They observe small-scale structures that they identify as a gravitational instability. In the present DNS study, we also observe overturning but caused by the nonlinear development of Kelvin-Helmholtz instabilities. This apparent discrepancy might be due to the influence of the Schmidt number or to the difference of the initial state. In particular, the vortices of the Lamb-Chaplygin dipole are closer together than those considered here. This is an issue that deserves further investigation.

The authors would like to thank Erik Lindborg for his suggestions on the article, as well as Geert Brethouwer for fruitful discussions on the parallelization of the code. This work is supported by IDRIS (CNRS) for computational facilities under project No. 61722.

\section{REFERENCES}

Billant, P. \& Chomaz, J.-M. 2000a Experimental evidence for a new instability of a vertical columnar vortex pair in a strongly stratified fluid. J. Fluid Mech. 418, 167-188.

Billant, P. \& Chomaz, J.-M. 2000b Theoretical analysis of the zigzag instability of a vertical columnar vortex pair in a strongly stratified fluid. J. Fluid Mech. 419, 29-63.

Billant, P. \& Chomaz, J. M. 2001 Self-similarity of strongly stratified inviscid flows. Phys. Fluids 13, 1645-1651.

Billant, P., Dritschel, D. G. \& Chomaz, J.-M. 2006 Bending and twisting instabilities of columnar elliptical vortices in a rotating strongly stratified fluid. J. Fluid Mech. 561, 73-102.

Brethouwer, G., Billant, P., Lindborg, E. \& Chomaz, J.-M. 2007 Scaling analysis and simulation of strongly stratified turbulent flows. J. Fluid Mech. 585, 343-368. 
Deloncle, A., Billant, P. \& Chomaz, J.-M. 2008 Three-dimensional stability of vortex arrays in a stratified and rotating fluid: theoretical analysis. J. Fluid Mech. (to be submitted).

Gill, A. E. 1982 Atmosphere-Ocean Dynamics. Academic.

Howard, L. N. 1961 Note on a paper of John W. Miles. J. Fluid Mech. 10, 509-512.

Laval, J.-P., McWilliams, J. C. \& Dubrulle, B. 2003 Forced stratified turbulence: successive transitions with Reynolds number. Phys. Rev. E 68, 036308.

LiLly, D. K. 1983 Stratified turbulence and the mesoscale variability of the atmosphere. J. Atmos. Sci. 40, 749-761.

LindBorg, E. 2006 The energy cascade in a strongly stratified fluid. J. Fluid Mech. 550, 207-242.

Miles, J. W. 1961 On the stability of heterogeneous shear flows. J. Fluid Mech. 10, 496-508.

Otheguy, P., Chomaz, J.-M. \& Billant, P. 2006 Elliptic and zigzag instabilities on co-rotating vertical vortices in a stratified fluid. J. Fluid Mech. 553, 253-272.

Riley, J. J. \& DeBruynKops, S. M. 2003 Dynamics of turbulence strongly influenced by buoyancy. J. Fluid Mech. 15, 2047-2059.

SiPP, D., JACQuin, L. \& Cossu, C. 2000 Self-adaptation and viscous selection in concentrated two-dimensional vortex dipoles. Phys. Fluids 12, 245-248.

Vincent, A. \& MeneguzZI, M. 1991 The spatial structure and statistical properties of homogeneous turbulence. J. Fluid Mech. 225, 1-20.

Waite, M. L. \& Bartello, P. 2004 Stratified turbulence dominated by vortical motion. J. Fluid Mech. 517, 281-308.

Waite, M. L. \& Smolarkiewicz, P. 2007 Instability and breakdown of a vertical vortex pair in a strongly stratified fluid. J. Fluid Mech. (submitted). 